Revue musicale OICRM

Du politique en analyse musicale, d'Esteban Buch, Nicolas

Donin et Laurent Feneyrou (dir.), Paris, VRIN, 2013, 256 pages

\title{
Judy-Ann Desrosiers
}

Volume 4, numéro 1, 2017

URI : https://id.erudit.org/iderudit/1040305ar

DOI : https://doi.org/10.7202/1040305ar

Aller au sommaire du numéro

Éditeur(s)

Observatoire interdisciplinaire de création et recherche en musique (OICRM)

ISSN

2368-7061 (numérique)

Découvrir la revue

Citer ce compte rendu

Desrosiers, J.-A. (2017). Compte rendu de [Du politique en analyse musicale, d'Esteban Buch, Nicolas Donin et Laurent Feneyrou (dir.), Paris, VRIN, 2013, 256 pages]. Revue musicale OICRM, 4(1), 165-170.

https://doi.org/10.7202/1040305ar

Ce document est protégé par la loi sur le droit d'auteur. L'utilisation des services d'Érudit (y compris la reproduction) est assujettie à sa politique d'utilisation que vous pouvez consulter en ligne.

https://apropos.erudit.org/fr/usagers/politique-dutilisation/ 


\section{Du politique en analyse musicale, d'Esteban Buch, Nicolas Donin et Laurent Feneyrou (dir.) Paris, VRIN, 2013, 256 pages}

\section{Judy-Ann Desrosiers}

Mots clés : analyse musicale ; histoire ; New Musicology ; politique ; recension.

Keywords: history; musical analysis; New Musicology; politics; review.

DU POLITIQUE

EN ANALYSE MUSICALE

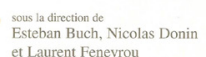

et Laurent Feneyrou

$\varepsilon$

VRIN
Cet ouvrage collectif, publié en 2013 chez Vrin et édité par Esteban Buch, Nicolas Donin et Laurent Feneyrou, s'inscrit dans la continuité de la discussion lancée lors d'un colloque tenu à l'Ircam en 2007 avec le soutien du CNRS. L'événement a été coorganisé par l'équipe "Musique » du Centre de recherches sur les arts et le langage (CRAL) dirigée par Buch, et par l'équipe «Analyses des pratiques musicales » (Ircam-CNRS) dirigée par Donin - et à laquelle est rattaché Feneyrou. Le collectif Du politique en analyse musicale s'inscrit également dans le sillage d'un précédent ouvrage, L'analyse musicale, une pratique et son histoire, dirigé par Rémy Campos et Donin, et paru en 2009 chez Droz.

Du politique en analyse musicale prend pour point de départ la discussion entamée lors du colloque de 2007 qui se proposait d'interroger les pratiques analytiques selon une approche historique. Le but était d'évaluer l'implication du politique en analyse musicale à travers deux grandes questions :

1. Qu'en est-il de la dimension politique des savoirs analytiques du passé et de ses prolongations dans les usages actuels des musicologues? 
2. Qu'en est-il du projet d'une pratique analytique contemporaine qui se donnerait comme objet précisément l'élucidation du lien entre musique et politique?

L'ouvrage recoupe ces deux questions. Cherchant ainsi à élucider le lien entre musique et politique relativement au travail analytique, la réponse qu'il tente d'esquisser vise à questionner les méthodes d'analyse actuelles et à mettre en évidence la dimension politique qui leur est inhérente.

Regroupant 14 articles, Du politique en analyse musicale est découpé en trois sections, respectivement intitulées "Enjeux de méthode " (p. 19-84), "Le politique à l'œuvre » (p. 85-165) et "Lectures politiques de l'analyse » (p. 169-252). Tous ces articles ne rencontrent pas nécessairement l'une ou l'autre des définitions du terme de "politique ». Comme le précisent les éditeurs en introduction, ce terme n'a pas servi à la répartition des articles. Ils ont plutôt fait le pari que la portée heuristique du concept de " politique » dépasserait la part d'indétermination qu'ils ont laissée quant à sa définition. Il aurait toutefois été éclairant de préciser davantage la nature du questionnement qui sous-tend chacune des trois sections proposées.

Quoi qu'il en soit, l'étude du politique en musique accorde nécessairement une place importante à l'étude du contexte entourant la production et la présentation d'une œuvre musicale, et ce peu importe la définition retenue. L'ouvrage s'inscrit donc directement dans la tendance de ce qu'on a appelé la New Musicology. Ce mouvement essentiellement actif dans les pays anglophones, a émergé d'abord aux États-Unis dans les années 1980, notamment avec les travaux de Joseph Kerman et Susan McClary, et s'est défini par le biais de nombreuses études qui déplaçaient le centre d'intérêt jusqu'alors axé sur l'étude de la partition musicale vers des analyses de nature historique, politique, culturelle ou sociale. Bien que $\mathrm{Du}$ politique en analyse musicale ne revendique pas d'appartenance directe à ce mouvement, la contribution de Lawrence Kramer et les nombreuses notes de bas de page renvoyant aux travaux des auteurs ayant marqué ce mouvement (par exemple, Rose R. Subotnik et Susan McClary) témoignent d'un partage d'idées important. La New Musicology est néanmoins considérée comme une tranche de l'histoire de la musicologie et les éditeurs du collectif Du politique en analyse musicale semblent vouloir montrer les études musicologiques qui ont été faites en ce sens dans les pays francophones.

\section{« ENJEUX DE MÉTHODE »}

En ce sens, la première section aborde des questions de méthodologie. Elle énonce, d'une part, les bases du débat qui a conduit à ce revirement académique étiqueté aujourd'hui de New Musicology, et fait état, d'autre part, des contributions de Kofi Agawu et Hermann Danuser, dont les études s'inspirent du modèle proposé par ce mouvement.

D'entrée de jeu, le but de l'analyse musicale est questionné, d'abord par une traduction inédite d'une conférence prononcée par Theodor Adorno en 1969 (« À propos du problème de l'analyse musicale », p. 19-31), qui avance l'idée que l'analyse d'une œuvre devrait chercher à expliquer les lois et les paradoxes que le compositeur 
a voulu forcer par la composition d'une œuvre (p. 27) ${ }^{1}$. Il convient de préciser qu'il ne s'agit là que d'une traduction de cette conférence et qu'aucune analyse critique du propos d'Adorno n'est proposée.

La section se poursuit par une traduction française, également inédite, d'une réflexion de Joseph Kerman publiée initialement en $1980^{2}$ ("Comment nous sommes entrés dans l'analyse, et comment en sortir», p. 33-52), à laquelle répond Agawu dans un article daté de $2004^{3}$ ("Comment nous sommes sortis de l'analyse, et comment y retourner », p. 53-70). Puisqu'elle repose d'abord sur le choix d'une œuvre, d'un style ou d'un genre musical jugé pertinent pour l'étude, l'analyse d'œuvres serait, selon Kerman, de nature idéologique. Pour l'auteur, cette manière de choisir l'œuvre à analyser constitue un obstacle insurmontable pour une Music Theory qui se targue pourtant d'être objective - a fortiori si l'on considère l'aspect mathématique de la Set Theory. Sa réflexion l'amène à revendiquer l'abandon du terme " analyse " pour celui de critique, lequel permet de mieux clarifier la part de subjectivité caractérisant cette activité. Près de 25 ans plus tard, la réponse d'Agawu met en perspective les propos de Kerman et soulève l'hétérogénéité des travaux musicologiques effectués depuis 1980. À l'heure actuelle, une New Musicology définie de manière unitaire fait encore défaut, et la diversité toujours croissante d'approches qui parviennent à rendre compte de la musique d'une manière pluridimensionnelle ne facilite pas la tâche. Toutefois, la Music Theory plutôt formaliste est mise à l'écart du projet de la New Musicology - c'est ce que Kerman souhaitait ; Agawu dénonce l'absence de cette discipline qui peut également jouer un rôle important dans le travail d'analyse de la musique. Cela dit, cette traduction du texte d'Agawu représente la seule contribution de ce collectif provenant d'un "music theorist». Cette particularité peut sans doute jouer en défaveur de l'ouvrage puisque la majorité des auteurs étant appelés à se prononcer sur des questions d'analyse ne sont pas spécialistes en Music Theory. Selon nous, ceci réduit la portée de l'ouvrage au sein de cette branche de la musicologie.

\section{« LE POLITIQUE À L'EEUVRE »}

Bien que la première section aborde des considérations d'ordre théoriques, la deuxième relève davantage de la pratique de l'analyse musicale et propose des études de cas portant sur le rôle de la (ou du) politique en musique à partir d'une œuvre. L'article de Yann Rocher (p. 131-145) aborde la question de la forme dans le Memorial to Lidice de Bohuslav Martinů en relation avec le souci de mémoire de la destruction

1 Conférence donnée par Adorno le 2 février 1969 à la Hochschule für Musik und Darstellende Kunst de Francfort. C'est la transcription de l'enregistrement, parue initialement dans Frankfurter Adorno Blätter (vol. 7, 2001, p. 73-89), qui a été traduite ici par Martin Kaltenecker.

2 Article paru initialement sous le titre " How We Got into Analysis, and How to Get out " dans Critical Inquiry (vol. 7, n 2, 1980, p. 311-331) et traduit ici de l'anglais par Martin Kaltenecker.

3 Agawu a présenté cette réflexion à la Faculté de musique de l'Université de Cambridge le 28 janvier 2004, dans le cadre des Donald Wort Lectures. Il s'agit ici d'une traduction française de Jean-Michel Magniez. Une version en anglais a été également publiée en 2004 dans Music Analysis (vol. 23, n 2-3, p. 267-286). 
du village tchèque de Lidice par les nazis en 1942. De son côté, Manuel Deniz Silva (p. 115-130) se penche sur les références politiques autour de la critique de la Rapsódia Portuguesa d'Ernesto Halffter. Ces deux articles rejoignent néanmoins une vision assez connue de l'étude du politique en musique qui fait référence à la période liée à la Seconde Guerre mondiale et à la violence présente sous les régimes totalitaires.

Dans la même section, Lawrence Kramer (p. 103-114) analyse la musique de Charles Ives afin de montrer que ses stratégies de composition traduisent une volonté de rompre avec les propositions de l'avant-garde européenne, dans un but manifestement nationaliste. L'article de Gianmario Borio (p. 147-165) aborde quant à lui la relation entre musique et cinéma en interrogeant la capacité de communication d'une œuvre lorsqu'elle est mise en relation avec des images. L'auteur s'intéresse spécifiquement à l'utilisation de la musique de Schubert dans le cinéma de Stanley Kubrick.

\section{« LECTURES POLITIQUES DE L’ANALYSE »}

La troisième et dernière section du livre explore le rapport politique-musique d'une manière plus abstraite, en interrogeant la place qu'occupe le politique à l'intérieur même de l'analyse, et en portant un regard plus spécifique sur ses méthodes et ses objectifs. Cette section vise ainsi à démontrer que l'analyse est toujours réalisée pour remplir un but précis. Le musicologue qui la pratique ne serait donc jamais complètement objectif et impartial : comme le soulignait déjà Kerman (p. 36), les choix inhérents au travail analytique induisent des biais que l'on ne doit pas sousestimer.

L'article de Maud Lambiet («Métamorphoses des politiques de l'analyse musicale à travers la Sonate en si mineur de Franz Liszt », p. 215-226) est peut-être le plus saisissant de ce point de vue. L'auteure compare 13 analyses, d'autant de théoriciens, de la Sonate en si mineur de Liszt afin de montrer la diversité des lectures de cette œuvre. La source de ces différences se trouve souvent dans les hypothèses de départ des musicologues, ce qui influence nécessairement l'approche analytique qu'ils adoptent. La comparaison des analyses met ainsi en lumière les raisons qui expliquent la variété des herméneutiques d'un même opus. À titre d'exemple, il semble inévitable que les analyses postulant l'existence d'un programme n'aboutissent pas au même résultat que celles qui ne reposent pas sur une telle hypothèse.

Les choix qui précèdent généralement toutes formes d'analyse opèrent également dans d'autres sphères de la musicologie. L'article d'Esteban Buch ("Was ist atonal ? Enjeux politiques de la définition de l'atonalisme, p. 169-181) questionne cette approche dans le processus de définition du concept d'atonalité, lié à la personnalité de Schönberg (d'ascendance juive) dans le contexte de l'Allemagne nazie. Buch examine comment le régime s'est positionné face à cette tendance d'avantgarde qui gagnait en adeptes, mais qu'il était difficile de légitimer comme orientation musicale du Troisième Reich. La contribution de Lothaire Mabru (" De la transcription en ethnomusicologie : outil d'analyse ou lit de Procuste? », p. 205-214) interroge ce que le chercheur peut modifier, voire abandonner lors du travail de transcription d'une musique qui utilise un système différent du sien, de même que les conséquences de cette pratique sur les études fondées sur des transcriptions. Enfin, l'article de 
Laurent Feneyrou ( Entre l'écorce et le bourgeon, trois analyses du refrain de la "Danse sacrale" », p. 227-252) montre que le politique est à l'œuvre aussi bien dans les méthodes, dans les conclusions que dans le but de l'analyse. Pour ce faire, trois analyses du refrain de la "Danse sacrale » du Sacre du printemps de Stravinsky sont mises en parallèle. Produites respectivement par Olivier Messiaen, Pierre Boulez et Jean Barraqué, chacune d'elles parvient à une conclusion différente. Pour Feneyrou, ce phénomène s'explique par le fait que chacun a cherché, par son analyse, à identifier des manières de composer correspondant à sa démarche compositionnelle.

$$
* * *
$$

Une des réserves importantes à propos de ce livre est l'absence d'une définition claire des termes " politique » et " analyse » qui y sont utilisés. La lecture des différents articles met en lumière une interprétation flexible de ces concepts, puisqu'ils recoupent des acceptions différentes d'un auteur à l'autre. Si les éditeurs se sont dégagés de l'obligation de définir le terme de "politique » en vue de laisser une plus grande liberté à leurs collaborateurs - ce qui n'est pas nécessairement à reprocher -, il aurait toutefois été judicieux et même indispensable que chaque auteur établisse clairement une définition opérationnelle du concept de politique véhiculé dans son texte. En effet, ce lien avec le (ou la) politique n'est pas toujours explicité au début des articles. Parfois, le terme n'apparaît qu'à la dernière phrase, ce qui est le cas de l'article de Martin Kaltenecker ("L'analyse considérée comme une guerre continuée par d'autres moyens. Remarques sur Heinrich Schenker, Milton Babbitt et la New Musicology", p. 183-204). La contribution est certes pertinente, puisqu'elle soulève plusieurs questions concernant la réception et l'assimilation de la théorie analytique de Schenker au sein des universités américaines, mais le lecteur doit sans cesse tenter de cerner la notion de politique entre les lignes du texte, ce qui rend la lecture difficile alors qu'une simple précision en introduction aurait éclairé l'argumentation de l'auteur. On constate aussi que ce qui est entendu par le terme " analyse » est plus ou moins précis et recoupe ainsi des acceptions différentes selon chaque collaborateur.

Néanmoins, certains explicitent dès le départ la référence au politique. Le texte d'Hermann Danuser ( "Musique et musicologie - en deçà et au-delà d'une codification politique», p. 71-84), par exemple, a le mérite de mettre de l'ordre dans la manière dont la musique se charge d'allusions politiques. La contribution de Rémy Campos («De la prise de note à l'analyse des musiques populaires sous la Troisième République ", p. 87-102), quant à elle, met à jour l'objectif nationaliste du projet d'analyse de la musique folklorique française à l'époque de la Troisième République. Enfin, 1'article signé par Manuel Deniz Silva s'intéresse à l'influence du contexte politique de la péninsule ibérique dans l'œuvre d'Ernesto Halffter.

Au-delà de cette critique autour de la définition du politique, il est pertinent de s'interroger sur la période historique couverte par les articles rassemblés dans ce collectif. Un examen rapide de la table des matières permet de constater que les sujets abordés se concentrent sur une période historique plutôt restreinte. En effet, les œuvres sur lesquelles repose l'argumentation des articles sont circonscrites de 1850 à 1950 , comme si la présence du ou de la politique en musique ne commençait véritablement qu'au XIX ${ }^{\mathrm{e}}$ siècle. 
À cet égard, la présence de textes concernant ce que l'on désigne généralement sous l'étiquette d'" écoles nationales "- espagnole, tchèque et américaine - dans la section sur "Le politique à l'œuvre ", semble associer le politique à cet éveil des nationalismes, lesquels sont caractéristiques du romantisme et de la fin du XIX siècle. Bien qu'on ne puisse nier l'importance de la politique durant cette période, les études s'intéressant à la notion de politique en musique sont peut-être encore trop circonscrites à cette période historique et à des régions qui se situent en marge $\mathrm{du}$ canon musical constitué des traditions germanique, française et italienne. Cette habitude d'aborder l'implication du politique en musique par le biais des nationalismes musicaux a trop souvent pour effet d'empêcher l'ouverture de ce champ de recherche à d'autres périodes historiques, et à d'autres régions et cultures musicales. Cette section de l'ouvrage ne semble donc pas exempte d'idéologie puisqu'elle ne concerne qu'une époque et une culture bien précise - celle de la culture occidentale de la fin du XIX $x^{e}$ siècle et de la première moitié $d u X X^{e}$ siècle. Pourtant, on pourrait soutenir sans crainte que le politique a toujours joué un rôle considérable dans l'humanité et qu'il a donc nécessairement influencé la musique à toutes les époques... Pourquoi alors aucun article ne s'interroge sur la dimension politique de la musique à la période baroque? Cette limitation temporelle est sans doute redevable aux champs de recherche privilégiés par les éditeurs du collectif, à savoir les musiques du Xxe siècle.

Selon nous, la plus grande faiblesse de cet ouvrage réside dans l'absence d'une conclusion faisant le point sur la diversité des perspectives qui sont abordées par les auteurs. Or, ce qui ressort de notre lecture globale est une remise en question du canon essentiellement issu de la culture germanique. Il est intéressant de constater à quel point le rejet de l'hégémonie allemande se dessine en arrière-plan des trois sections de l'ouvrage. La première plaide pour un renouvellement d'une musicologie encore trop attachée à la manière dont elle a été institutionnalisée au courant du XIX ${ }^{e}$ siècle en Allemagne. La deuxième interroge les références au politique dans un contexte fortement influencé par la montée du nazisme. Finalement, la dernière section porte une réflexion sur l'influence du politique dans la définition des concepts de la musicologie, de même que sur ses approches et ses outils, pour la plupart développés par la culture germanique.

Paru en 2013, soit 30 ans après l'apparition de la New Musicology, Du politique en analyse musicale semble vouloir mettre en lumière l'apport considérable que ce mouvement a eu sur la diversification du discours sur la musique. La juxtaposition des 14 articles tend à démontrer l'enrichissement de la musicologie depuis ce renouvellement méthodologique apparu dans les universités américaines vers les années 1980. En réalisant un état des lieux de l'étude du politique en musique à travers un ensemble d'articles à première vue hétérogène, il nous semble que les éditeurs invitent le lecteur à garder à l'esprit la nécessité d'une remise en question de la discipline et de ses méthodes. En somme, le foisonnement de perspectives offert par la diversité des contributions ouvre la discussion sur la manière d'aborder une œuvre et le discours sur la musique en général. 